Symposium on the EPIIEMIOLOGY OF LEPROSY, Geilo, Norway, 1981

Lepr Rev (198I) 52, Suppl I, 77-83

\title{
Leprosy - a zoonosis
}

\section{GP WALSH, WM MEYERS, CH BINFORD, PJ GERONE, RH WOLF \& JR LEININGER}

Armed Forces Institute of Pathology, Washington, D.C. 20306; Delta Regional Primate Research Center, Covington, LA 70433; University of Minnesota, St. Paul, MN

The traditional view is that humans are the only reservoir of Mycobacterium leprae and that leprosy is transmitted only by leprosy patients. In the last seven years we have studied naturallyacquired leprosy in three different animal species: nine-banded armadillos (Dasypus novemcinctus), a chimpanzee, and a mangabey monkey. These discoveries demonstrate conclusively that naturallyacquired leprosy is not confined to humans and that animal reservoirs of the disease must be considered.

Naturally-acquired indigenous leprosy in wild-caught armadillos

BACKGROUND AND PRESENT STATUS

Investigators at Gulf South Research Institute, New Iberia, Louisiana, discovered in 1974 the first armadillo with indigenous leprosy in a recently-captured armadillo. The only gross pathologic change in this animal was enlarged inguinal lymph nodes. Smears of these nodes contained large numbers of acid-fast bacilli (AFB). At necropsy there was disseminated infection by a non-cultivable Mycobacterium. This infection was histopathologically like the experimental infection that follows the inoculation of $M$. leprae in the armadillo, including the invasion of peripheral nerves by acid-fast bacteria $(\mathrm{AFB})$. In the ensuing twelve months, seven armadillos captured from three different locations in southern Louisiana were detected with the disease and, as with the first animal, the etiologic agent was indistinguishable from M. leprae (1). Subsequent examination of tissues from 1033 armadillos showed rates of infectivity in Louisiana ranging from $4 \%$ to $29.6 \%$ (2).

We have now found a total of 80 armadillos with indigenous leprosy. 79 were captured from 12 locations in Louisiana and one was from a wildlife preserve in northeast Texas. Most of the positive sites in Louisiana are swampy with dense foliage and harbor large numbers of insects.

Ten of the 80 infected armadillos had gross lesions when cap- 
tured - typically large subcutaneous nodules. Naturally-infected armadillos have been reported independently by three other centers. Smith et al at the University of Texas reported the disease in two armadillos captured in southern Louisiana (3). Two armadillos with indigenous leprosy have come from near College Station, Texas, one was detected in the San Diego Zoo in California (4) and the second was captured by a team from the Center for Disease Control, Atlanta, Georgia (5).

\section{IDENTIFICATION OF THE ETIOLOGIC AGENT}

The etiologic agent of naturally-acquired leprosy in armadillos was identified using the criteria outlined in 1968 at the 8 th International Leprosy Congress in London, with the addition of more recently developed supplemental tests to assist in the identifiaation

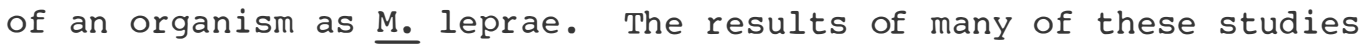
have been published in detail $(6,7)$ and will only be summarized here:

1. Histopathology: Macrophages contain large numbers of AFB frequently arranged in packets or globi and stain more intensely with the Fite-Faraco than with the Ziehl Neelsen methods. The globi and invasion of peripheral nerves by AFB are similar to that seen in leprosy in man.

2. Microbiology: Etiologic agent is not cultivable although positive cultures of organisms of the Mycobacterium avium-intracellulare complex were obtained from the lymph nodes of eight animals with disseminated infections. Acid-fastness of the etiologic agent was pyridine extractable.

3. Immunology: Lepromin prepared from tissues of naturallyinfected armadillos and standard human lepromin gave similar reactions in leprosy patients; fluorescent antibody studies and immunodiffusion studies show the natural agent to be indistinguishable from M. leprae.

4. Animal passage: Normal armadillos inoculated with the natural agent developed disseminated leprosy (8). The growth patterns of selected isolates in the mouse footpad were like M. leprae.

5. Electronmicroscopy: Freeze-etch and thin section preparations revealed no difference between tissues from armadillos with the natural infection and those with experimental leprosy (9).

\section{ORIGIN AND SIGNIFICANCE OF INDIGENOUS LEPROSY IN ARMADILLOS}

Armadillos have not been domesticated making it unlikely that they acquired the disease directly from lepromatous patients, however, in rural areas contact with contaminated fomites e.g., clothing and dressings must be considered. Multibacillary patients living at home in the pre-sulfone era could have provided a ready source of viable M. leprae. Leprosy bacilli are known to remain viable for up to 
seven days in dried nasal secretions from leprosy patients (10). Because armadillos commonly encounter insects and soil, these must also be considered as modes for transmission.

Once introduced into an armadillo population, animal to animal transfer of leprosy either by direct contact or inhalation could have resulted in the disease becoming endemic in this species. In addition, we have detected $M$. leprae in the mammary glands of lactating armadillos and consider transmission via mother's milk as a distinct possibility (8) .

Leprosy in humans has been endemic in Louisiana for more than 150 years (11). The armadillo migrated into the state in 1926, therefore, armadillos are not responsible for the initial introduction of leprosy into Louisiana. A study by the Center for Disease Control failed to show any association between human leprosy patients in Louisiana and contact with armadillos (12). Recently, however, lepromatous leprosy was diagnosed in a man who in Texas had killed and dressed many armadillos (13). We believe, therefore, that armadillos could serve as a source of infection for humans. The frequency and distribution of leprosy in armadillos requires that this animal species be considered a potential source of leprosy in epidemiologic considerations for humans in the United States.

Spontaneous leprosy in a chimpanzee

\section{BACKGROUND}

Naturally-acquired leprosy in a non-human primate, a chimpanzee, was first reported in 1977 by Donham and Leininger (14). The animal was obtained by the University of Iowa from an import company who had purchased it from African nationals in Sierra Leone. Two months after arrival at the University of Iowa, the chimpanzee was inoculated, along with seven other chimpanzees, with bovine leukemia virus. Two months later macules began to appear on the skin of the animal and progressed over the entire trunk and limbs and the ears became markedly thickened within six months. A biopsy specimen of a nodule on the ear revealed diffuse infiltrations of macrophages containing large numbers of $\mathrm{AFB}$. In the succeeding 14 months, lesions developed on the lower lip, nares, eyebrows and scrotum. Peripheral nerves showed invasion by AFB. Nasal smears contained large numbers of AFB.

33 months after the appearance of the initial skin lesions, the animal died following administration of anesthesia for a routine procedure. Necropsy revealed disseminated leprosy involving the liver, spleen, lymph node, testes, lungs, eyes and nasal mucosa (15). Microbiological studies revealed that the AFB in the tissues of this chimpanzee were identical to M. leprae (16). 


\section{SOURCE AND SIGNIFICANCE}

As with the armadillo, we speculate that this chimpanzee acquired the infection from human leprosy patients. We have been told that in some African countries chimpanzee mothers are killed and the young chimpanzees are hand-raised by natives until they are sold to animal exporters. Leprosy is highly endemic in Sierra Leone. Thus, there is a possibility that this chimpanzee came in close contact with an individual or individuals with untreated lepromatous disease prior to shipping to the United States. The possibility that infection occurred after arrival in the United States is extremely remote.

We have not been able to establish any direct relationship between the inoculation of bovine leukemia virus and the development of leprosy. However, there was serological evidence that all of the chimpanzees were infected by the virus. The experimental viral infection thus could have altered the immune competence of the animal, permitting a latent $M$. leprae infection to progress. Because the frequency of naturally-acquired leprosy in wild chimpanzees is unknown, we are not able to determine the potential role of this species in the epidemiology of leprosy in humans.

Because chimpanzees are found throughout the tropical rain forests of Africa where there are many leprosy-endemic areas, the knowledge that naturally-acquired leprosy can occur in this species is important. However, the discovery of naturally-acquired leprosy in a chimpanzee suggests that the susceptibility of this species should be re-examined using inocula containing large numbers of viable M. leprae (e.g. from armadillos).

Naturally-acquired leprosy in a mangabey monkey (Dercocebus torquatus atys)

\section{BACKGROUND}

This animal, commonly known as the "sooty" mangabey monkey, was captured in Nigeria and imported to the United States about 1975. The earliest lesions of leprosy were firm nodules on the face, first seen in September 1979. Four months later, following a histopathologic diagnosis of lepromatous leprosy (AFIP \# 1724396) the animal was transferred from Gulf South Research Institute, New Iberia, Louisiana, to the Delta Regional Primate Research Center, Covington, Louisiana. At that time, the face was heavily infiltrated and ulcerated. There was nodular thickening of the ears and over the extensor surface of the forearms.

Peripheral nerves were not enlarged and there were no paralytic deformities. The results of identification studies of the etiologic agent have been published (17). The microbiologic, immunologic, and electron microscopic studies showed that the infecting organism was 
indistinguishable from Mycobacterium leprae. Histopathologically, the diagnosis was subpolar lepromatous leprosy. Infiltration of dermal nerves was a frequent finding.

\section{PARALYTIC DEFORMITIES}

16 months after the cutaneous lesions were first observed, paralytic deformities developed in this animal. These consisted of paralysis of the intrinsic muscles of the foot and the hand and of the extensor and peroneal muscles of the foot with accompanying deformities. To our knowledge, this is the first leprosy-infected animal that has manifested paralytic deformities simulating those seen in human leprosy.

\section{RESPONSE TO TREATMENT}

Soon after the deformities were observed, the general health of the animal began to deteriorate and treatment was initiated. Rifampin (RFM) was given at a dosage of $10 \mathrm{mg} / \mathrm{kg}$ per os for a period of 28 days. Following one week of RFM treatment, the general health of the animal was markedly improved. We attributed much of the initial response to the effect of RFM on bacterial infections secondary to extensive ulcerations of the skin, particularly those of the face and extremities. Although the results of the viability studies in the mouse footpad are not yet available, histopathologic evaluation of biopsies taken two weeks after initiating therapy showed that virtually all of the AFB in the tissues were irregularly stained. Following treatment with RFM, DADDS therapy was initiated at a dosage of $20 \mathrm{mg}$ every 77 days.

\section{PASSAGE STUDIES IN NORMAL MANGABEY MONKEYS}

Suspensions of AFB from the naturally-infected mangabey monkey were inoculated intravenously and subcutaneously into two normal mangabeys. One of the animals developed lesions at inoculation sites on the ear four months after inoculation. 17 months after inoculation, progressive lesions involving areas remote from inoculation sites (e.g. scrotum) have developed. Histopathologically, these lesions were like those in the initial monkey. In the second mangabey, inoculated at the same time, the infection has progressed much more slowly and histopathologically the disease in this animal more closely resembles borderline leprosy. Thus, our early observations suggest individual differences in host response indicating that a spectrum of leprosy similar to that seen in man may develop in mangabey monkeys.

Suspensions of M. leprae freshly harvested from an armadillo with advanced disseminated leprosy were inoculated into two normal 
mangabey monkeys. One of the animals developed distinct lesions at several of the inoculation sites in the skin four months after inoculation. Biopsies of these lesions revealed an active infection that was histopathologically similar to early lepromatous leprosy. The second animal also developed lesions at the inoculation sites but clinically and histopathologically the infection appears to be developing more slowly than in the first animal. Nine months postinoculation, there is active infection in both animals.

\section{SIGNIFICANCE OF SPONTANEOUS LEPROSY IN A MANGABEY MONKEY}

The discovery of naturally-acquired lepromatous leprosy in a mangabey monkey augments the evidence already provided by nine-banded armadillos and the chimpanzee that $M_{0}$ leprae can spontaneously cause lepromatous leprosy in non-human species. While it is reasonable to suggest that this animal may have become infected following contact with an untreated lepromatous patient, the possibility that it had contact with a lepromatous mangabey in an African forest cannot be excluded. Of perhaps even greater significance is the potential that the mangabey monkey may have as a subhuman primate model for leprosy: Because monkeys are phylogenetically closely related to man, studies of the disease in this species may provide data that are highly relevant to leprosy in man. Moreover, the development of paralytic deformities and the early results of passage studies suggesting a spectrum of the disease could make the mangabey model the most complete model for the study of leprosy.

\section{Summary}

We have observed naturally-acquired leprosy in three animal species: nine-banded armadillos, a chimpanzee, and a mangabey monkey. The frequency of the infection in armadillos in the southern United States provides sufficient evidence to allow designation of the armadillo as a reservoir for the disease in this area. Although the prevalence of the infection of chimpanzees and mangabey monkeys in the wild is not known, the existence of spontaneous leprosy in these species requires that they be given consideration in the epidemiology of leprosy in geographic areas inhabited by these animals. The infection found in all three species has been of the lepromatous or near-lepromatous type and therefore highly bacilliferous and contagious. The role that these species play in the transmission of leprosy to man must now be ascertained.

\section{References}

Walsh GP, Storrs EE, Burchfield HP, Cottrell E, Vidrine MF, Binford $\mathrm{CH}$. Leprosy-like disease occurring naturally in arma- 
dillos. J Reticuloendothel Soc, 1975, 18, 347-51.

2 Walsh GP, Storrs EE, Meyers WM, Binford $\overline{\mathrm{CH}}$. Naturally-acquired leprosy-like disease in the nine-banded armadillo (Dasypus novemcinctus): Recent epizootiologic findings. J Reticuloendothel Soc, 1977, 22, 363-67.

3 Smith JH, File SK, Nagy BA, Folse DS, Buckner JA, Webb LJ, Beverding AM. Leprosy-like disease of wild armadillos in French Acadiana, Louisiana. J Reticuloendothel Soc, 1978, 24, 705-19.

4 Anderson JM, Benirschke K. Personal communication.

5 Center for Disease Control Veterinary Public Health Notes, October 1978 .

6 Binford $\mathrm{CH}$, Meyers WM, Walsh GP, Storrs EE, Brown HL. Naturallyacquired leprosy-like disease in the nine-banded armadillo (Dasypus novemcinctus): Histopathologic and microbiologic studies of tissues. J Reticuloendothel Soc, 1977, 22, 377-88.

7 Meyers WM, Walsh GP, Brown HL, Rees RJW, Convit J. Naturallyacquired leprosy-like disease in the nine-banded armadillo (Dasypus novemcinctus): Reactions in leprosy patients to lepromins prepared from naturally infected armadillos. J Reticuloendothel Soc, 1977, 22, 369-75.

8 Meyers WM, Binford $\mathrm{CH}$, Brown HL, Walsh GP. Leprosy in wild armadillos. Proceedings of Symposium on Comparative Pathology of Zoo Animals, National Zoological Park, Smithsonian Institution Press, 1980, 543-49.

9 Marchiondo AA, Smith JH, File SK. Naturally-occurring leprosylike disease of wild armadillos: Ultrastructure of lepromatous lesions. J Reticuloendothel Soc, 1980, 27, 311-25.

10 Davey $\mathrm{TF}$, Rees RJW. The nasal discharge in leprosy. Clinical and bacteriological aspects. Lepr Rev, 1972, 45, 121-34.

11 Feldman RA, Sturdivant M. Leprosy in Louisiana, 1855-1980: An epidemiologic study of long term trends. Am J Epid 1975, 102, 303-21.

12 Filice G. Lack of observed association between armadillo contact and leprosy in humans. Am J Trop Med Hyg, 1977, 26, 137-42.

13 Freiberger HG, Fudenberg H. An appetite for armadillo. Hospital Practice, 1981, 137-44.

14 Donham KJ, Leininger JR. Spontaneous leprosy-like disease in a chimpanzee. J Inf Disease, 1977, 132-36.

15 Leininger JR, Donham KJ, Meyers WM. Leprosy in a chimpanzee. Postmorten lesions. Int J Lepr, 1980, 48, 414-21.

16 Leininger JR, Donham KJ, Rubino MJ. Leprosy in a chimpanzee. Morphology of the skin lesions and characterization of the organism. Vet Pathol, 1978, 15, 339-46.

17 Meyers WM, Walsh GP, Brown HL, Fukunishi Y, Binford CH, Gerone PJ, Wolf RH. Naturally-acquired leprosy in a mangabey monkey (Cercocebus sp.). Int J Lepr, 1980, 48, 495-96. 